\title{
RISCOS AO FETO EM GESTANTES INFECTADAS POR COVID-I9
}

\author{
RISKS TO THE FETUS IN PREGNANT WOMEN INFECTED BY COVID-I9
}

\author{
Wesley Pereira de Freitas ${ }^{1}$ \\ Bruna Lorena Andrade de Melo Maia ${ }^{2}$ \\ Elaine Barbosa Menezes ${ }^{3}$ \\ Gabriel Barbosa Menezes ${ }^{4}$ \\ Davi de Oliveira Menezes ${ }^{5}$ \\ Julio Cesar Ponte 6 \\ Leticia Deola Vieira ${ }^{7}$ \\ Leonardo Deola Vieira ${ }^{8}$ \\ Maria Emília Bezerra ${ }^{9}$ \\ Roberto de Barros Silva ${ }^{10}$
}

RESUMO: Introdução: a gravidez é o processo biológico decorrente da fecundação entre o óvulo feminino (ovócito) pelos espermatozoides, tal fenômeno ocorre dentro do útero materno após a ejaculação. Aas gestantes estarem passando por mudanças em seus corpos, e tudo isso acaba alterando o sistema fisiológico, imunológico e principalmente o cardiopulmonar, as gestantes acabam sendo suscetíveis a complicações respiratórias e sistémicas decorrentes de infecções por algum tipo de vírus, e isso não é diferente para o covid-ı. Objetivo: abordar através de uma revisão da literatura de forma sistemática, quais são os riscos que um feto pode sofrer quanto a gestante apresenta um quadro de infecção por Covd-19. Metodologia: A metodologia usada foi através de uma Revisão da Literatura, com os seguintes bases da dados utilizados foi a Biblioteca Virtual de Saúde (BVS). Bases de Dados em Enfermagem (BDENCE); Medical Literature Analysis and Retrieval System Online (MEDLINE; Literatura Latino-Americana e do Caribe em Ciências da Saúde (LILACS); Índice Bibliográfico Español en Ciencias de la Salude (IBECS) e Scientific Electronic Library Online (SciELO), onde foi possível selecionar os artigos completos que descrevesse na integra sobre a influência do covid-ı́ no feto em período gestacional.

\footnotetext{
${ }^{1}$ Acadêmico de Medicina, Unifacs - Salvador, BA.

${ }^{2}$ Acadêmica de Medicina, Unifacs - Salvador, BA.

${ }^{3}$ Acadêmica de Medicina, Unifacs - Salvador, BA.

4 Acadêmico de Medicina, Unifacs - Salvador, BA.

${ }^{5}$ Acadêmico de Medicina, Unifacs - Salvador, BA.

6 Acadêmico de Medicina, Unifacs - Salvador, BA.

7 Acadêmica de Medicina, Unifacs - Salvador, BA.E-mail: leticiadeolavieira@gmail.com

${ }^{8}$ Acadêmico de Medicina, Unifacs - Salvador BA.

9 Acadêmica de Medicina, Unifacs - Salvador, BA. E-mail: emiliaz.8.bezerra@gmail.com

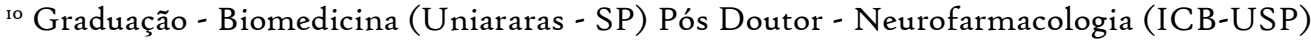

Doutor - Neurofarmacologia (FCFRP-USP) e pela Medicine School at King's College London, Guys Campus (Inglaterra) Mestre - Patologia Experimental e Farmacologia - (FMRP -USP)

Docente do curso de medicina da Universidade de Salvador (UNIFACS).E-mail: roberto.barros@unifacs.br
} 
Resultados: foi possível selecionar 300 artigos divididos em 6 bases de dados, desse total apenas 16 forma selecionados para a obtenção dos dados. Conclusão: A infecção pelo CovidI9 trás vários problemas a saúde gestacional das mães, pode ocasionar no aumento de partos prematuros, abortos espontâneos, problemas respiratórios no recém-nascido por serem assintomáticos dificultando o diagnóstico rápido e preciso do vírus, aumentado da internação nas Unidades de terapia intensiva e semi-intensiva e o aumento dos óbitos fetais.

Palavras-chave: Covid-19. Gravidez. Materno-fetal. Riscos.

ABSTRACT: Introduction: pregnancy is the biological process resulting from fertilization between the female egg (oocyte) by sperm, such a phenomenon occurs within the maternal uterus after ejaculation. Aspregnant women are experiencing changes in their bodies, and all this ends up altering the physiological, immunological, and especially cardiopulmonary system, pregnant women end up being susceptible to respiratory and systemic complications resulting from infections by some type of virus, and this is not different for covid-19. Objective: approach through a systematic review of the literature, what are the risks that a fetus may suffer when the pregnant woman presents a picture of Covid-I9 infection. Methodology: The methodology used was that described by Laurence Ganong (1987) as a study byIntegrative Literature Review(RIL), with the use of the Virtual Health Library (VHL) where the Nursing Databases (BDENCE) are indexed; Medical Literature Analysis and Retrieval System Online (MEDLINE), Latin American and Caribbean Literature on Health Sciences (LILACS), Bibliographic Index Español en Ciencias de la Salude (IBECS) and Scientific Electronic Library Online (SciELO), where it was possible to select the complete describing articles in full the influence of covid-I9 in the fetus in gestational period. Results: it was possible to select 300 articles divided into 6 databases of this total only 16 forms selected to obtain the data. Conclusion: Covid-I9 infection causes several problems in the gestational health of mothers, can lead to the increase in premature births, spontaneous abortions, respiratory problems in the newborn because they are asymptomatic, making it difficult to diagnose the virus quickly and accurately, increased hospitalization in intensive and semi-intensive care units and increased fetal deaths.

Keywords: Covid-ı. Pregnancy. Maternal-fetal. Risks.

\section{INTRODUÇÃO}

$\mathrm{O}$ ano de 2019 teve início com uma pandemia causada pelo novo coronavírus 2 (SARS-Cov-2). Vírus esse que causa grave infecção das vias respiratórias e dependendo do quadro do paciente poderá levá-lo óbito repentinamente. SARS-Cov-2 teve sua origem na cidade de Wuhan na China, e teve sua publicação quanto calamidade pública no dia II de março de 2020 quando já tinha alcançado cerca de aproximadamente 292.I42, totalizando cerca de 195 países que já estavam sendo afetados pela pandemia (RAMALHO, 2020). 
O coronavírus foi descrito pela primeira vez no ano de 1996 pelos médicos e pesquisadores Tyrell e Bynoem (FAUCI et al., 2020). Suas pesquisas estavam sendo realizadas através dos cultivos do vírus coletados de pacientes que apresentavam apenas um resfriado comum, mas quando foram realizar testes in vitro perceberam que o coronavírus tinha um grau de letalidade maior que o vírus da gripe (VELAVAN, 2020).

Os vírus pertencentes a família dos coronavírus são chamados de emergenciais e infectam rapidamente o ser humano (AHMAD et al., 2020). Pacientes infectados por covidI9 desenvolvem a Síndrome respiratória aguda grave coronavírus 2 (SARS-Cov-2), e apresentam sintomas que são classificados como leves, moderador ou gravíssimos, podendo aumentar os números de óbitos. Apesar de alguns pacientes estarem infectados pelo covid19, eles não apresentam nenhum sintoma característico da doença sendo chamados de assintomáticos (CIOTTI et al., 2020).

A infecção por covid-ıg acontece em todas as faixas etárias, tendo uma maior porcentagem em homens adultos acima dos 40 anos e apresentem comorbidade. Já nas mulheres o percentual é menor, contudo, o percentual muda quando estão acometidas por comorbidade (SOUZA et al., 2020). Mulheres gestantes infectadas por covid-I9 podem sofrer com alterações no desenvolvimento do feto em todas as fases de seu desenvolvimento gestacional (DE ALBUQUERQUE et al., 2020).

Observando a atual situação da infecção por covid-ı́ e o aumento de mulheres grávidas devido ao isolamento social bem como a preocupação com a saúde de mãe e bebê, este trabalho tem como objetivo principal abordar através de uma revisão da literatura de forma sistemática, quais são os riscos que um feto pode sofrer quanto a gestante apresenta um quadro de infecção por covd-ı. E, com isso debater quais seriam as medidas de atenção que gestantes e bebês precisam para que não haja prejuízo graves a saúde de ambos.

\section{METODOLOGIA}

O estudo trata-se de uma revisão da literatura e tem como objetivo realizar a investigação de artigos científicos referentes ao tema proposto e que estejam disponíveis nas plataformas digitais, livros, revistas etc. O método escolhido foi uma revisão integrativa que tem com proposta principal organizar de forma sistemática os dados encontrados nos artigos, que possa facilitar o entendimento e a compreensão dos resultados. 
A pesquisa iniciou-se no mês de setembro de 202I, a coleta dos dados foi feita nas plataformas Biblioteca Virtual de Saúde (BVS); Bases de Dados em Enfermagem (BDENCE); Medical Literature Analysis and Retrieval System Online (MEDLINE), Literatura Latino-Americana e do Caribe em Ciências da Saúde (LILACS), Índice Bibliográfico Español en Ciencias de la Salude (IBECS) e Scientific Electronic Library Online (SciELO), mediante ao uso das palavras-chaves covid-ı, gravidez, materno-fetal e riscos.

Quanto aos artigos, foram incluídas publicações completas referente aos anos de 2020 e 2021 que tinham como temática covid-19 e gravidez nos idiomas português, inglês e espanhol. Como critério de exclusão foi utilizado para desconsiderar artigos com anos de publicações inferiores a 2020 e 2021, artigos semelhantes que estavam publicados em plataformas diferentes. Os artigos selecionados foram tabulados em planilha do Excel 2019 possibilitando a extração dos dados de interesse, e com isso fosse possível responder os objetivos deste trabalho.

\section{RESULTADOS E DISCUSSÃO}

A pesquisa feita nas bases de dados Biblioteca Virtual de Saúde (BVS); Bases de Dados em Enfermagem (BDENCE); Medical Literature Analysis and Retrieval System Online (MEDLINE), Literatura Latino-Americana e do Caribe em Ciências da Saúde (LILACS), Índice Bibliográfico Español en Ciencias de la Salude (IBECS) e Scientific Electronic Library Online (SciELO), possibilitou encontrar um total de 300 artigos disponíveis (figura I).

Com a leitura dos artigos na integra foi possível a exclusão de 130 trabalhos que apresentavam duplicidade em base de dados diferentes. Após essa exclusão por duplicidade restaram 170 artigos, de onde foram excluídos 94 por não aprofundavam sobre os problemas que a infecção poderia causar em mães e fetos. Aprofundando a avaliação dos artigos, deixando legíveis 76 artigos. Contudo uma nova avaliação dos 76 artigos acerca dos resultados obtidos, percebeu-se que 6o não estavam completos sobre informações sobre o covid-19 e de como ele afetaria a gestante. 
Figura I. Fluxograma da seleção dos estudos

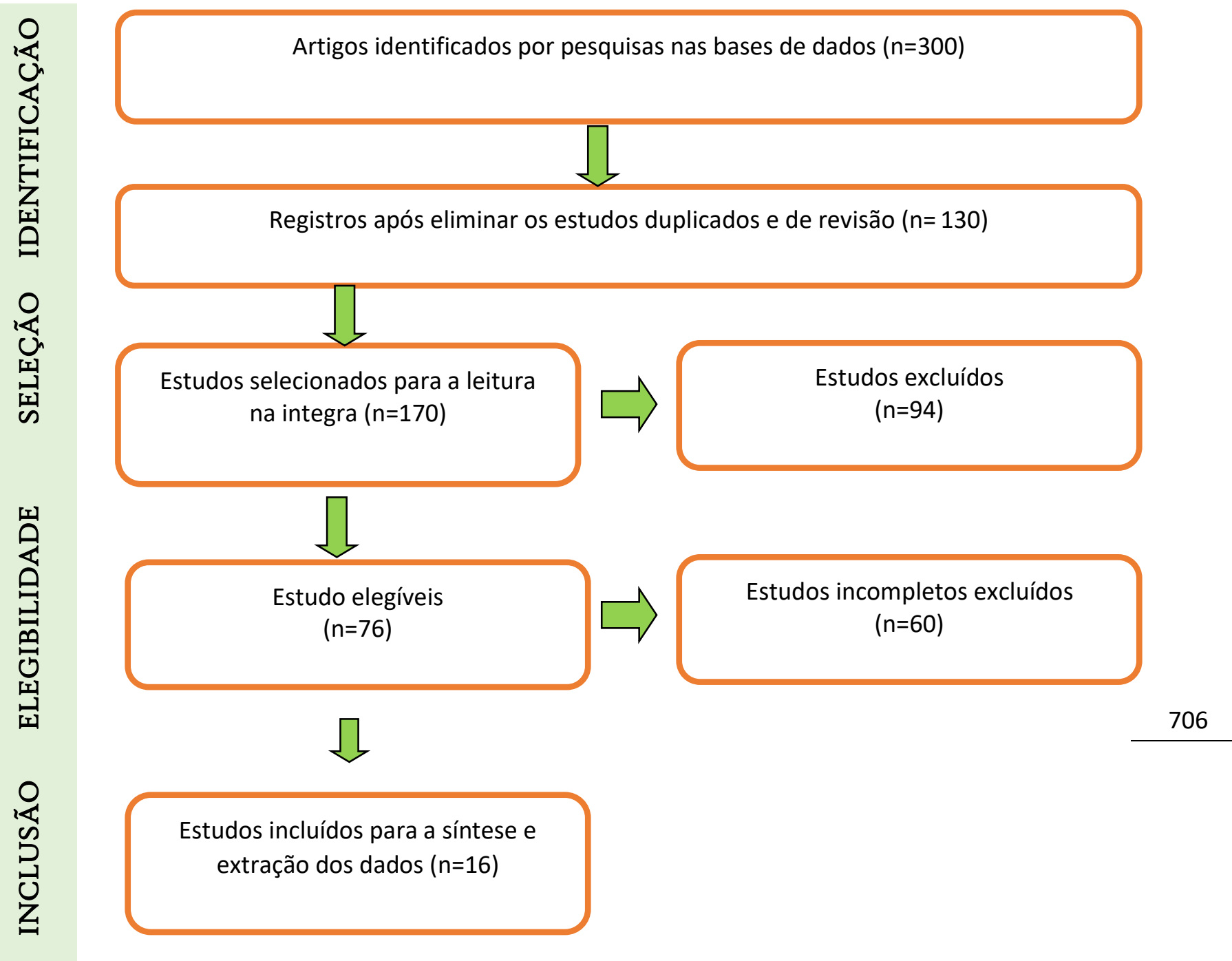

Fonte: MONTEIRO et al., 2019 adaptado.

Mediante a isso foram incluídos neste trabalho 16 artigos que respondiam de forma clara sobre quais problemas o covid-I9 poderia trazer as grávidas e como a infecção pode prejudicar a saúde do feto no decorrer da gravidez caso a mãe esteja infectada. Para a construção da tabela I foram incluídos os dados: título da obra, autor, ano de publicação do artigo e a base de dados onde eles foram encontrados. 
Quadro I. Relação dos artigos selecionados com destaque para título, autor, ano de publicação e base de dados em qual foi encontrado.

\begin{tabular}{|c|c|c|c|c|}
\hline № & Título da obra & Autor & $\begin{array}{l}\text { Ano de } \\
\text { publicação }\end{array}$ & $\begin{array}{l}\text { Base de } \\
\text { dados }\end{array}$ \\
\hline OI & $\begin{array}{l}\text { COVID-i9 na gravidez, o que } \\
\text { sabemos? }\end{array}$ & Ramalho & 2020 & SCIELO \\
\hline 02 & The COVID-I9 epidemic & Velavan & 2020 & MEDLINE \\
\hline 03 & $\begin{array}{lll}\text { Covid-I9 } & \text { Navegando } & \text { no } \\
\text { desconhecido. }\end{array}$ & Fauci et al. & 2020 & LILACS \\
\hline 04 & COVID-rg: Aspectos zoonóticos & Ahmad et al. & 2020 & SCIELO \\
\hline 05 & $\begin{array}{l}\text { Revisões críticas em ciências de } \\
\text { laboratório clínico }\end{array}$ & Ciotti et al. & 2020 & SCIELO \\
\hline o6 & $\begin{array}{l}\text { Reflexões em tempos de COVID-ı: } \\
\text { diferenciais por sexo e idade. }\end{array}$ & Souza et al. & 2020 & SCIELO \\
\hline 07 & $\begin{array}{l}\text { Implicações da COVID-ı9 para } \\
\text { pacientes gestantes }\end{array}$ & $\begin{array}{l}\text { De } \\
\text { Albuquerque } \\
\text { et al. }\end{array}$ & 2020 & MEDLINE \\
\hline o8 & $\begin{array}{l}\text { Assistência às gestantes e recém- } \\
\text { nascidos no contexto da infecção } \\
\text { COVID-ıg: uma revisão sistemática }\end{array}$ & Rondelli et al & 2020 & LILACS \\
\hline 09 & $\begin{array}{l}\text { Pregnant women with COVID- I9 } \\
\text { and its consequences for newborns }\end{array}$ & Vieira et al & 202I & BVS \\
\hline IO & $\begin{array}{l}\text { Avaliação de risco dos medicamentos } \\
\text { usados para o tratamento de Covid-ı9 } \\
\text { em gestantes infectadas e transmissão } \\
\text { vertical }\end{array}$ & $\begin{array}{l}\text { Fernandes et } \\
\text { al }\end{array}$ & 2020 & MEDLINE \\
\hline II & $\begin{array}{l}\text { Assistance to twins at risk of } \\
\text { congenital COVID-ig infection, } \\
\text { experience report }\end{array}$ & Moreira et al & 2020 & BVS \\
\hline 12 & $\begin{array}{l}\text { Gravidez na adolescência, iniciação } \\
\text { sexual e gênero: perspectivas em } \\
\text { disputa. }\end{array}$ & $\begin{array}{l}\text { Brandão e } \\
\text { Cabral }\end{array}$ & 2020 & LILACS \\
\hline 13 & $\begin{array}{l}\text { COVID-I9: Um Novo Desafio para a } \\
\text { Cardiopatia na Gravidez }\end{array}$ & Avila et al & 2020 & LILACS \\
\hline I4 & $\begin{array}{l}\text { Covid-ı9 na gravidez, parto e pós- } \\
\text { parto imediato: implicações e } \\
\text { intercorrências }\end{array}$ & $\begin{array}{l}\text { Boaventura et } \\
\text { al }\end{array}$ & 2021 & BVS \\
\hline 15 & $\begin{array}{l}\text { Repercussões fetais e possíveis } \\
\text { complicações da COVID-I9 durante } \\
\text { a gestação. }\end{array}$ & Ramiro & 2020 & LILACS \\
\hline I6 & $\begin{array}{l}\text { Gestação múltipla no surto de SARS- } \\
\text { CoV-2: o desafio do pré-natal }\end{array}$ & $\begin{array}{l}\text { Santana e } \\
\text { Elito }\end{array}$ & 2020 & SCIELO \\
\hline
\end{tabular}


No período gestacional a mulher sofre diversas mudanças endócrinas, psicológicas e sociais. E, isso contribui com a queda do sistema imunológico, facilitando a infecção de diversas enfermidades inclusive a infecções causadas pelo covid-ı9 (DE SOUZA FARIAS et al., 2020; DI OLIVEIRA et al., 202I).

Covid-ı9 está sendo um dos vírus que tem causados inúmeras complicações em mulheres gestantes, sendo necessário cuidados intensivos no atendimento das gestantes. Grávidas infectadas por covd-ı tem maior chance de partos prematuros. Já para os bebês que chegam a nascer com vida apresentam problemas de desenvolvimento tanto físico, psicológico, motor e respiratórios, podendo levar a óbito (BHERING et al., 202I).

Segundo Santos et al. (202I) e Silva et al. (202I), as gravidas estão incluídas no grupo de risco para covid-ı, devido ao aumento da morbimortalidade que elas podem apresentar. Quando estão infectadas pelo covid-ı9 podem apresentar sintomas de resfriados leves, tosse seca e febre. Já nos casos mais graves as elas podem apresentar sintomas de fadiga, dispneia, diarreia, congestão nasal e coriza, problemas respiratórios como síndrome respiratória aguda grave (SARS), e todos esses fatores comprometem a saúde do feto em desenvolvimento.

Vieira et al. (202I) que gestantes infectadas pelo covid-I9 tem mais chances de apresentar pré-eclâmpsia, sofrimento fetal e parto prematuro. Já no caso dos recém-nascido quando infectados através da transmissão vertical não apresentam sintomas comuns da doença, com isso dificultando o diagnóstico afim de evitar problemas respiratórios causados pelos vírus.

Boaventura et al. (202I) reforçam, que apesar de algumas gestantes serem assintomáticas ou apresentarem sintomas leves de gripes, tem aumentado a ocorrência de partos prematuros através da realização de cesáreas de emergências. Pois mães com covidI9 tem apresentado estados graves da doença, que comprometem as vias respiratórias, prejudicando a saúde do feto.

Quanto ao feto de mães gravida infectadas pelo covd-r9, ZAKARIA et al (2020), destaca que essas pacientes devem ser monitoradas de acordo com os protocolos relacionados ao pré-natal e todos os exames devem ser realizados com o intuído de verificar a saúde do feto, pois o covid-ı9 pode ocasionar problemas na gestação ou até mesmo levar a morte por abortos espontâneos. 
Dessa forma para prevenir problemas com o feto em mães acometidas pelo covid-I9 Santana e Elito (2020) e Rondelli et al. (2020) recomendam a realização de ultrassonografias a cada 14 dias após o final da doença de acordo com o protocolo de Royal College of Obstetricians and Gynaecologists (RCOG), para que possa ser observado anomalias devido a infecção pelo vírus. Pois mulheres gravidas infectadas por covid-ı́ estão propensas a complicações decorrente da infecção que vão desde a parto pré-maturo espontâneo, complicações das vias respiratórias dos bebês e até mesmo mortalidade perinatal e fetais.

Avila e Carvalho (2020) reforçam o cuidado com as gestantes cardiopatas infectadas pelo covid-ı, devido a falta de terapia específica para o tratamento da covid-ı para esse grupo, são necessários que a forma de prevenção seja mais eficaz para o cuidado de gestantes e feto. E, que devem ser acompanhadas por uma equipe multidisciplinar para otimizar o acompanhamento da gestante evitando com isso problemas para o feto. Pois as graves consequências da covid-ıو somadas as complicações decorrentes a infecção pelo vírus mais a cardiopatia ou hipertensão arterial trará um desfecho materno incerto.

Fernandes et al. (2020) e Moreira et al. (2020) afirmam que as gravidas estão dentro do grupo de risco, não só pela facilidade de infecção por covid-ı9, mas também pela dificuldade do tratamento medicamentoso que elas não podem ser submetidas por causa do feto em desenvolvimento. Pois os medicamentos para o tratamento são fortes e podem comprometer o desenvolvimento fetal podendo ocasionar o aborto espontâneo. Os autores enfatizam sobre a importância de ser utilizados protocolos efetivos e seguros frente a possíveis riscos teratogênicos, em que drogas não testadas podem acarretar ao desenvolvimento saudável do feto.

\section{CONCLUSÃO}

A infecção de gestante pelo covid-ı9 tem chamado a atenção dos pesquisadores. Por estarem incluídas no grupo de risco devido apresentarem comorbidades e fragilidade do sistema imunológico pela exigência do feto. A infecção pelo covid-ı́ trás vários problemas a saúde gestacional das mães, pode ocasionar no aumento de partos prematuros, abortos espontâneos, problemas respiratórios no recém-nascido por serem assintomáticos dificultando o diagnostico rápido e preciso do vírus, aumentado da internação nas Unidades de terapia intensiva e semi-intensiva e o aumento dos óbitos fetais. 
Dessa forma é necessário que sejam usadas medidas de prevenção já estabelecidas pelo Ministério da Saúde para as gestantes. Sendo necessário o isolamento das mães e bebês no período de infecção e proporcionar um atendimento multidisciplinar as grávidas, com o intuito de diminuir as percas gestacionais e problemas posteriores decorrentes da infecção das mães e fetos pelo vírus.

\section{REFERÊNCIAS}

AHMAD, T.; KHAN, M.; HUI, J.; BONILlA-ALDANA, K.; RODRIGUEZMORALES, A. J. Covid-ı: Aspectos zoonóticos. Medicina de viagem e doenças infecciosas, v. 36, p. 101607, 2020.

AVILA, W. S.; CARVAlHO, R. C. D. Covid-I9: Um Novo Desafio para a Cardiopatia na Gravidez. Arq Bras Cardiol. IIS(I):1-4, 2020.

BHERING, N; B. V.; ARNDT, C. G., FILHO, D. A. D. P. G. O parto prematuro induzido pela Covid-ı: uma revisão da literatura. Brazilian Journal of Health Review, v. 4, n. 2, p. 440I-4415, 2021.

BOAVENTURA, M. D.; COSTA, M. R.; NUNES, R. N.; SANTO CARINE, C. S. C. S.; SANTOS, S.; SAMPAIO, I. L.; MOURA, L. R. Covid-i9 na gravidez, parto e pós-parto imediato: implicações e intercorrências. Brazilian Journal of Development, 7(7), 202I.

CABRAL, C. da S.; BRANDÃO, E. R. Gravidez na adolescência, iniciação sexual e gênero: perspectivas em disputa. Cadernos de Saúde Pública, v. 36, p. eoooz9420, 2020.

CIOTTI, M.; CICCOZZI, M.; TERRINONI, A.; JIANG, W.; WANG.; BERNARDINIÍ, S. A pandemia Covid-ı. Revisões críticas em ciências de laboratório clínico, v. 57, n. 6, pág. 365-388, 2020.

DE AlbuQUerque, L. P.; MONTE, A.V. L.; DE ARAÚJO, R. M. Implicações da COVID-19 para pacientes gestantes. Revista Eletrônica Acervo Saúde, v. I2, n. Io, p. e4632e4632, 2020. 
DE OLIVEIRA, F. P.; DA SILVA LIMA, M. R.; DE FARIAS, F. L. R. Assistência à saúde de gestantes no contexto da pandemia do COVID-I9. Revista Interdisciplinar, v. I4, n. I, p. 15, 2021.

DE SOUZA FARIAS, L. M et al. Gestação e cuidados: atenção à saúde psíquica da mulher em tempos de COVID-19. 2020.

FAUCI, A. S.; LANE, H. CLIFFORD, R.; ROBERT, R. Covid-I9 navegando no desconhecido. N Engl J Med. 2020.

FERNANDES, T. A.; VENTUROTTI, T. M.; DE SOUZA CARVAlHO, F. R. Avaliação de risco dos medicamentos usados para o tratamento de Covid-19 em gestantes infectadas e transmissão vertical. Unesc em Revista, 4(2), II-I9, 2020.

MOREIRA, I. F.; TENÓRIO, J. L.; DE FARIAS MOREIRA, R. T.; CALUMBY, R. J. N.; DE LIMA, C. M. F.; DE SOUSA, E. C. A. Assistência a gemelares com risco de infecção congênita por COVID-ı9, relato de experiência. Research, Society and Development, 9 (II), 2020.

RAMALHO, C. Covid-ı9 na gravidez, o que sabemos?. Acta Obstétrica e Ginecológica Portuguesa, v. I4, n. I, p. 6-7, 2020.

RAMIRO, N. C. M. P. et al. Repercussões fetais e possíveis complicações da COVID-ı9 durante a gestação. Saúde Coletiva (Barueri), v. ıo, n. 54, p. 2679-269o, 2020.

RONDELLI, G.; JARDIM, D.; HAMAD, G.; LUNA, E.; MARINHO, W.; MENDES, L.; GRATÃO, L. Assistência às gestantes e recém-nascidos no contexto da infecção COVIDI9: uma revisão sistemática. Desafios-Revista Interdisciplinar da Universidade Federal do Tocantins, 7 (Especial-3), 48-74, 2020.

SANTANA, E. F. M.; ELITO, J. Gestação múltipla no surto de SARS-CoV-2: o desafio do pré-natal. Einstein (São Paulo), I8. ;18: I-2, 2020. 
SANTOS, M. S.; DE FREITAS, A. L. G. C.; DE LIMA, T. H. B. Os efeitos materno-fetais da covid-I9 no período gestacional. Brazilian Journal of Health Review, v. 4, n. 5, p. 190671908I, 2021.

SILVA, L. T et al. Gestação e pandemia da COVID-I9: Impactos no binômio maternofetal. Research, Society and Development, v. io, n. 7, p. e23510716416-e23510716416, 2021.

SOUZA, L. G.; RANDOW, R.; SIVIERO, P. C. L. Reflexões em tempos de COVID-I9: diferenciais por sexo e idade. Com Ciências Saúde [Internet], v. 31, p. 75-83, 2020.

VELAVAN, T. P.; MEYER, C. G. The Covid-ı9 epidemic. Medicina tropical e saúde internacional, v. 25, n. 3, pág. $278,2020$.

VIEIRA, A. R. L. de C.; ROCHA, A. J. C.; FARIA, A. L. O. de; OLIVEIRA, R. R. A. de.; BARROS, G. B. S. Pregnant women with COVID- I9 and its consequences for newborns. Research, Society and Development, [S. 1.], v. Io, n. I2, p. 202I.

ZAKARIA, O. M., DAOUD, M. Y. I., ALKHALAF, G. I., ALBSHR, F. A., ALJARRASH, K. M., AlShEEF, N. J., ALQAHTANI, N. H. Feto-maternal and neonatal care during COVID-I9 pandemic: A perceptual study from Saudi Arabia. Sapporo MJ, v. 54, n. 7, p. I7,2020 